\title{
Random Measure Algebras Under O-dot Product and Morse-Transue Integral Convolution
}

\author{
Jason Hong Jae Park \\ Correspondence: Jason Hong Jae Park, Department of Mathematical Sciences, University of Nevada, Las Vegas, Las \\ Vegas, NV, USA. E-mail: jason.park@unlv.edu
}

Received: October 8, 2019 Accepted: November 5, 2019 Online Published: November 14, 2019

doi:10.5539/ijsp.v8n6p73

URL: https://doi.org/10.5539/ijsp.v8n6p73

\begin{abstract}
In this article, we consider two operations of random measures: O-dot product and the convolution product by MorseTransue integral. With these two operations, we construct algebras of random measures. Also we investigate further on the explicit forms of the products of Wiener processes by O-dot operation and by Morse-Transue integral convolution.
\end{abstract}

Keywords: random measure, convolution, probability, algebra

\section{Introduction}

Convolution is a very interesting subject in classical analysis. The convolution product is of interest to us since the operation is often closed in the same class. This allows us to build an algebra structure. One of the classical examples is $L^{2}$ space. Let $(X, \mathcal{M}, \mu)$ be a measurable space and let $L^{2}(\mu)$ be be the set of all measurable function $f: X \rightarrow \mathbb{C}$ such that $\int|f|^{2} d \mu<\infty$. The convolution of $f, g \in L^{2}$ is defined by $f * g(x)=\int f(x-y) g(y) d y . f * g$ is also in $L^{2}$, therefore $L^{2}$ is an algebra. $L^{2}$ is also a complete normed space, therefore it is a Banach algebra. A Banach algebra is well-studied area of functional analysis. By constructing algebra structure of $L^{2}$ functions, the study of functional analysis has expanded since we can apply thousands of algebra theorems. Then we can ask if similar things can be done in probability, in other words, 'Can we build an algebra of stochastic processes?' If this can be done, it will enrich the study of statistics and probability.

To build an algebra structure, we need two operations. For instance, we have the addition and multiplication in real numbers $\mathbb{R}$. In $L^{2}$, the first operation is addition. If we add $f, g \in L^{2}$ then $f+g \in L^{2}$ also. However, if we choose the second operation as multiplication, $f \cdot g$ is not necessarily in $L^{2}$. Therefore, we need another operation, and that is the convolution.

Defining the convolution is the key to build an algebra. In the past, some researchers have defined the convolution of stochastic processes with restricted conditions or something related to stochastic processes. J.E. Huneycutt (1972) has introduced a convolution of vector measures, and D. Dehay (1991) has shown the product of two $L^{p}$-harmonizable series is harmonizable. C. Graham and B. Schreiber (1984) defined a convolution of bilinear forms and built an algebra of bilinear forms. M.M. Rao (2012) extended a convolution of bilinear forms to a convolution of random measures, and J.H.J. Park (2016) constructed an algebra of second ordred random measures by using the convolution of M.M. Rao.

In this ariticle, we build two more algebras by using operation introduced from M.M. Rao (2012), namely, O-dot product $\odot$ and the convolution by strict Morse-Transue integral. Section 2 mainly consists of preliminary results and definitions of O-dot product and strict Morse-Transue integral. In section 3 and 4, we build the algebras of random measures by using O-dot product and strict Morse-Transue integral, respectively.

Remark 1 H.S. Chung, D. Skoug and J. Chang (2014) introduced a convolution product on Wiener Space. Their convolution is the convolution of nonrandom functionals on a Wiener Space which is a vector space of nonrandom integrable functionals relative to the Wiener measure which is translation invariant in an infinite dimensional space $\mathbb{R}^{\infty}$. However, there is no randomness involved in the functions. It is an interesting functional analysis problem, but our interest is in the convolution of stochastic processes and measures of various types, and is distinct from their works.

\section{Preliminaries}

\subsection{Random Measures and Bimeasures}

We introduce definitions of random measures and bimeasures.

Definition 2.1 Let $(G, \mathcal{G})$ be a measurable space, where $G$ is a locally compact abelian group and $\mathcal{G}$ is $\sigma$-algebra of $G$. Let $Z$ be a vector-valued $\sigma$-additive set function such that $Z: \mathcal{G} \rightarrow L^{2}(\Omega, \Sigma, P)$, where $(\Omega, \Sigma, P)$ is a probability measure space. Then $Z$ is termed a random measure.

The domain of a random measure is a $\sigma$-algebra $\mathcal{G}$, which is a family of sets $\mathcal{G} \subset \mathcal{P}(G)$ that is closed under complements 
and countable unions.

In this article, we focus on the second order random measure. An ouput of $Z$ is a random variable with second moment. If the range of $Z$ is $L^{p}$-space, then $Z$ would be a $p$ th order random measure.

Definition 2.2 Let $(G, \mathcal{G})$ be a measurable space, where $G$ is a locally compact abelian group and $\mathcal{G}$ is $\sigma$-algebra of $G$.

1. A mapping $\beta: \mathcal{G} \times \mathcal{G} \rightarrow \mathbb{C}$ is called a bimeasure if it is separately additive, that is, if $\beta(A, \cdot)$ and $\beta(\cdot, B)$ are (scalar valued) additive measures for every $A, B \in \mathcal{G}$.

2. A bimeasure $\beta: \mathcal{G} \times \mathcal{G} \rightarrow \mathbb{C}$ is said to be $\sigma$-additive if it is separately $\sigma$-additive.

3. A bimeasure $\beta: \mathcal{G} \times \mathcal{G} \rightarrow \mathbb{C}$ is said to have a bounded or finite Vitali variation if

$$
|\beta|=\sup \left\{\sum_{i=1}^{n} \sum_{j=1}^{n}\left|\beta\left(E_{i}, F_{j}\right)\right|: E_{i}, F_{j} \in \mathcal{G}\right\}<\infty,
$$

where all $E_{i}$ 's are disjoint and all $F_{j}$ 's are disjoint.

4. A bimeasure is called positive definite if

$$
\sum_{i=1}^{n} \sum_{j=1}^{n} a_{i} \overline{a_{j}} \beta\left(A_{i}, B_{j}\right) \geq 0
$$

for all $a_{i}, a_{j} \in \mathbb{C}, A_{i}, B_{j} \in \mathcal{G}$ and $1 \leq i, j \leq n$ where $n \in \mathbb{N}$.

A bimeasure is an 'analog' of a covariance function. The following lemma states that there is always a corresponding bimeasure $\beta$ to $Z$ and vice versa. In this article, if a bimeasure $\beta$ corresponds to the random measure $Z$ then we denote $Z \sim \beta$.

Lemma 2.3 From Rao (2012) Let $(G, \mathcal{G}$ be a measurable space, where $G$ is locally compact abelian group and $\mathcal{G}$ is a $\sigma$-algebra of bounded Borel sets of $G$, and $\beta: \mathcal{G} \times \mathcal{G} \rightarrow \mathbb{C}$ be a bounded bimeasure. Then it is positive definite if and only if there is a probability space $(\Omega, \Sigma, P)$ and a random measure $Z: G \rightarrow L^{2}(P)$ inducing the bimeasure in the sense that $\beta(A, B)=\langle Z(A), Z(B)\rangle_{L^{2}(P)}, A, B \in \mathcal{G}$.

The inner product in $L^{2}(P)$ is defined by $\langle X, Y\rangle_{L^{2}}=\int_{\Omega} X \bar{Y} d P$ where $X, Y \in L^{2}(P)$. Therefore $\langle Z(A), Z(B)\rangle_{L^{2}(P)}=$ $\int_{\Omega} Z(A) \overline{Z(B)} d P=E[Z(A) \overline{Z(B)}]$.

Lemma 2.4 From Park (2015) Let $\alpha \in \mathbb{C}, Z: \mathcal{G} \rightarrow L^{2}(\Omega, \Sigma, P)$ be a random measure, and $\beta$ be the corresponding bimeasure of $Z$, in other words, $\beta(A, B)=E[Z(A) \overline{Z(B)}]$. Then $\alpha Z$ has the corresponding bimeasure $|\alpha|^{2} \beta$ (i.e. If $Z \sim \beta$ then $\alpha Z \sim|\alpha|^{2} \beta$ )

Proof Let $\beta^{\prime}$ be the corresponding bimeasure of $\alpha Z$. Then $\beta^{\prime}(A, B)=E[\alpha Z(A) \bar{\alpha} \overline{Z(B)}]=|\alpha|^{2} E[Z(A) \overline{Z(B)}]=|\alpha|^{2} \beta(A, B)$.

Lemma 2.4 will be useful in section 3.

\subsection{Space of Random Measures and Bimeasures}

Let $R M(G)$ and $B M(G)$ be the space of random measures and bimeasures, respectively. Then we have the following elementary results from J.H.J. Park (2016).

Theorem 2.5 From Park (2016)

1. $B M(G,+)$ is an abelian group.

2. $B M(G,+)$ is a unitary $\mathbb{C}$-module.

3. $R M(G,+)$ is an abelian group.

4. $R M(G,+)$ is a unitary $\mathbb{C}$-module.

\subsection{O-dot Product}

The following proposition motivates the concept. 
Proposition 2.6 From Rao (2012) Let $\beta_{i}: \mathcal{G}_{i} \times \mathcal{G}_{i} \rightarrow \mathbb{C}, i=1,2$ be a pair of positive definite kernels and $\beta=\beta_{1} \cdot \beta_{2}:$ $\left(\mathcal{G}_{1} \times \mathcal{G}_{1}\right) \times\left(\mathcal{G}_{2} \times \mathcal{G}_{2}\right) \rightarrow \mathbb{C}$ as their pointwise product. Then $\beta$ is positive definite. If we let $\mathbb{H}_{\beta}, \mathbb{H}_{\beta_{1}}, \mathbb{H}_{\beta_{2}}$ the corresponding reproducing kernel Hilbert (or Aronszajn) spaces, then $\mathbb{H}_{\beta}=\mathbb{H}_{\beta_{1}} \otimes \mathbb{H}_{\beta_{2}}$, so that $\mathbb{H}_{\beta}$ is a tensor product of $\mathbb{H}_{\beta_{1}}$ and $\mathbb{H}_{\beta_{2}}$.

The proof of the Proposition 2.6 is detailed in Rao (2012). The Proposition 2.6 leads to the following definition.

Definition 2.7 From Rao (2012) Let $(G, \mathcal{G})$ be a measurable space and $Z_{i}: \mathcal{G} \rightarrow L_{0}^{2}(P)$ be a pair $(i=1,2)$ of random measures into $L_{0}^{2}(P)$ the Hilbert space of (equivalence classes of) centered (complex) random variables on a probability space $(\Omega, \Sigma, P)$ with covariance bimeasures $\beta_{i}: \mathcal{G} \times \mathcal{G} \rightarrow \mathbb{C}$ given by $\beta_{i}(A, B)=<Z_{i}(A), Z_{i}(B)>$ using the inner product notation. Let $\beta=\beta_{1} \cdot \beta_{2}:(\mathcal{G} \times \mathcal{G}) \times(\mathcal{G} \times \mathcal{G}) \rightarrow \mathbb{C}$ be the product, pointwise as in Proposition 2.6.

Definition 2.7 leads to the definition of O-dot product of random measures $Z_{1}$ and $Z_{2}$, where $Z_{1}, Z_{2}$ are corresponding random measures of bimeasures $\beta_{1}, \beta_{2}$, respectively. This is well illustrated in M.M. Rao (2012). However, we will slightly alter the definition of O-dot product from Rao's text since the problem with pointwise product occurs immediately. In fact, the product $\beta_{1} \cdot \beta_{2}$ does not produce the bimeasure of the same class. The dimension of domain gets larger as we multiply. We will define a new O-dot product with a modification and build an algebra in section 3 .

\subsection{Convolution of Bimeasures}

The integration of bimeasures by M. Morse and W. Transue (1956) is necessary to define the convolution of bimeasures.

Definition 2.8 If $\left(\Omega_{i}, \Sigma_{i}\right), i=1,2$ are measurable spaces and $(\Omega, \Sigma)$ is their product (so $\Omega=\Omega_{1} \times \Omega_{2}$ and $\left.\Sigma=\Sigma_{1} \otimes \Sigma_{2}\right)$, $f_{i}: \Omega_{i} \rightarrow \mathbb{C}$ (measurable relative to $\left.\Sigma_{i}, i=1,2\right)$ are given, then the pair $\left(f_{1}, f_{2}\right)$ is said to be strictly $\beta$-integrable where $\beta$ is a bimeasure on $\Sigma_{1} \times \Sigma_{2}$, provided the following two conditions hold:

1. $f_{1}$ is $\beta(\cdot, B)$-Lebesgue integrable for each $B \in \Sigma_{2}$ and $f_{2}$ is $\beta(A, \cdot)$-Lebesgue integrable for each $A \in \Sigma_{1}$ such that $\tilde{\beta_{1}}(A, F)=\int_{F} f_{2}\left(\omega_{2}\right) \beta\left(A, d \omega_{2}\right)$ is $\sigma$-additive in $A \in \Sigma_{1}$ for each $F \in \Sigma_{2}$ and $\tilde{\beta_{2}}(E, B)=\int_{E} f_{1}\left(\omega_{1}\right) \beta\left(d \omega_{1}, B\right)$ is $\sigma$-additve in $B \in \Sigma_{2}$ for each $E \in \Omega_{1}$.

2. $f_{1}$ is $\tilde{\beta_{1}}(\cdot, F)$-Lebesgue integrable, $f_{2}$ is $\tilde{\beta_{2}}(E, \cdot)$-Lebesgue integrable and

$$
\int_{E} f_{1}\left(\omega_{1}\right) \tilde{\beta_{1}}\left(d \omega_{1}, F\right)=\int_{F} f_{2}\left(\omega_{2}\right) \tilde{\beta_{2}}\left(E, d \omega_{2}\right) \quad E \in \Sigma_{1}, F \in \Sigma_{2} .
$$

The common value in above is denoted by $\int_{E} \int_{F}\left(f_{1}, f_{2}\right) d \beta=\int_{E} f_{1}\left(\omega_{1}\right) \tilde{\beta_{1}}\left(d \omega_{1}, F\right)=\int_{F} f_{2}\left(\omega_{2}\right) \tilde{\beta_{2}}\left(E, d \omega_{2}\right)$.

The following proposition also can be considered as a definition. $\mathcal{B}([0,1])$ is a $\sigma$-algebra of Borel subsets of $[0,1]$.

Proposition 2.9 From Rao (2012) Let $Z_{i}: \mathcal{B}([0,1]) \rightarrow L^{2}(P), i=1,2$ are a pair of random measures, and let $\beta_{i}$ 's are corresponding bimeasures of $Z_{i}$ 's, with finite Vitali variations. Then the convolution of $\beta_{1}$ and $\beta_{2}$ are defined by

$$
\left(\beta_{1} * \beta_{2}\right)(A, B)=\int_{0}^{1} \int_{0}^{1} \beta_{1}(A-x, B-y) \beta_{2}(d x, d y), A, B \in \mathcal{B}([0,1]),
$$

where the integration is strict Morse-Transue integral. Also, $\left(\beta_{1} * \beta_{2}\right)(\cdot, \cdot)$ is a well-defined positive definite bimeasure on $\mathcal{B}([0,1]) \times \mathcal{B}([0,1])$ and there is a random measure $Z: \mathcal{B}([0,1]) \rightarrow L^{2}(P)$ whose bimeasure is $\left(\beta_{1} * \beta_{2}\right)(\cdot, \cdot)$.

Existence of such $Z$ is guaranteed by Lemma 2.3. The covolution of bimeasures allows to us define the convolution of random measures, since there is one-to-one correspondence between bimeasures and random measures.

2.5 Wiener Process

A Wiener process is one of examples of stochastic processes.

Definition 2.10 A process $\left\{W(t), t \in \mathbb{R}^{+}\right\}$is called a Wiener process with a positive diffusion coefficient $\sigma$ if

1. Each increment $W(s+t)-W(s)$ is $N\left(0, \sigma^{2} t\right)$

2. For every pair of disjoint time intervals $\left(t_{1}, t_{2}\right],\left(t_{3}, t_{4}\right]$ with $0 \leq t_{1}<t_{2} \leq t_{3}<t_{4}$, the increments $W\left(t_{4}\right)-W\left(t_{3}\right)$ and $W\left(t_{2}\right)-W\left(t_{1}\right)$ are independent random variables, and similarly for $n$ disjoint time intervals, where $n$ is an arbitrary positive integer.

3. $W(0)=0$ and $W(t)$ is continuous as a function of $t$.

Let $\{W(t)\}_{t \in \mathbb{R}^{+}}$be a Wiener Process and $\mathcal{G}$ be a $\sigma$-algebra of Borel subsets of $\mathbb{R}^{+}$. Pick $A=(t, s) \in \mathcal{G}$, where $0 \leq t<s \leq \infty$. Then we define a random measure $Z: \mathcal{G} \rightarrow L^{2}(\Omega, \Sigma, P)$ by $Z(A)=W(s)-W(t)$. This is an example of a stochastic process which can be written in terms of a random measure. 
Definition 2.11 Given a centered $L^{2}(P)$-stochastic process $\left\{X(t): t \in \mathbb{R}^{+}\right\}$, its covariance function, or kernel is given by $C(t, s)=\operatorname{Cov}(X(t), X(s))$.

Lemma 2.12 From Park (2015) For the Wiener Process $\left\{W(t): t \in \mathbb{R}^{+}\right\}$, the covariance funtion is $\operatorname{Cov}(W(s)$, $W(t))=$ $\sigma^{2} \min \{s, t\}$ for $s, t \geq 0$.

Proof. Recall $E[W(t)]=0$, and $E[W(t)]^{2}=\sigma^{2} t$. Then for $0 \leq s<t$,

$$
\begin{aligned}
\operatorname{Cov}(W(s), W(t)) & =E[W(s) W(t)] \\
& =E(W(s)(W(t)-W(s)+W(s))] \\
& =E[W(s)(W(t)-W(s))]+E[W(s) W(s)] \\
& \text { Since } W(s) \text { and } W(t)-W(s) \text { are independent } \\
& =E[W(s)] E[W(t)-W(s)]+E\left[W^{2}(s)\right] \\
& =0+E\left[W^{2}(s)\right] \\
& =\sigma^{2} s .
\end{aligned}
$$

Similarly, for $0 \leq t<s$, we obtain $\operatorname{Cov}(W(s), W(t))=\sigma^{2} t$, which leads to the formula

$$
\operatorname{Cov}(W(s), W(t))=\sigma^{2} \min \{s, t\} \text { for } s, t \geq 0
$$

\section{O-dot Product and Convolution of Bimeasures}

\subsection{O-dot Product}

The product $\beta=\beta_{1} \cdot \beta_{2}$ in Definition 2.7 does not have the same domain as $\beta_{1}, \beta_{2}$, therefore $\beta$ is not in the same class as $\beta_{1}, \beta_{2}$. Recall the diagonal set of $\mathbb{R} \times \mathbb{R}$ is $\{(x, x) \in \mathbb{R} \times \mathbb{R} \mid x \in \mathbb{R}\}$, which is isomorphic to $\mathbb{R}$. We extend this idea to $(\mathcal{G} \times \mathcal{G}) \times(\mathcal{G} \times \mathcal{G})$. Let the diagonal set of $(\mathcal{G} \times \mathcal{G}) \times(\mathcal{G} \times \mathcal{G})$ be $\{(A \times B, A \times B) \mid A \times B \in \mathcal{G} \times \mathcal{G}\}$. Now, consider the following definition. Let $\tilde{\beta}:(\mathcal{G} \times \mathcal{G}) \times(\mathcal{G} \times \mathcal{G}) \rightarrow \mathbb{C}$ be such that

$$
\tilde{\beta}= \begin{cases}\beta & \text { on diagonal set of }(\mathcal{G} \times \mathcal{G}) \times(\mathcal{G} \times \mathcal{G}) \\ 0 & \text { otherwise. }\end{cases}
$$

$\tilde{\beta}$ is defined on isomorphic copy of $\mathcal{G} \times \mathcal{G}$. We rewrite

$$
\tilde{\beta}(A, B)=\beta(A \times B, A \times B) .
$$

Now we assert that the $\tilde{\beta}$ is positive definite and $\sigma$-additive.

Lemma 3.1 Suppose $\tilde{\beta}$ is defined as above.

1. $\tilde{\beta}$ is positive definite

2. $\tilde{\beta}: \mathcal{G} \times \mathcal{G} \rightarrow \mathbb{C}$ is separately $\sigma$-additive.

Proof

1. $\tilde{\beta}$ is positive definite since $\beta$ is positive definite by Proposition 2.6.

2. We consider the disjoint set $(A, C)$ and $(B, D)$. Let $\tilde{\beta}$ be the pointwise product of $\beta_{1}, \beta_{2} \in B M(G)$, and $A, B, C, D \in \mathcal{G}$, where $A \cap C=\varnothing, B \cap D=\varnothing$. Then

$$
\begin{aligned}
& \tilde{\beta}((A \cup C),(B \cup D)) \\
& =\beta((A \cup C) \times(B \cup D),(A \cup C) \times(B \cup D)) \\
& =\beta((A \times B) \cup(A \times D) \cup(C \times B) \cup(C \times D), \\
& (A \times B) \cup(A \times D) \cup(C \times B) \cup(C \times D)) \\
& =\beta(A \times B, A \times B)+\beta(A \times B, A \times D)+\beta(A \times B, C \times B)+\beta(A \times B, C \times D) \\
& +\beta(A \times D, A \times B)+\beta(A \times D, A \times D)+\beta(A \times D, C \times B)+\beta(A \times D, C \times D) \\
& +\beta(C \times B, A \times B)+\beta(C \times B, A \times D)+\beta(C \times B, C \times B)+\beta(C \times B, C \times D) \\
& +\beta(C \times D, A \times B)+\beta(C \times D, A \times D)+\beta(C \times D, C \times B)+\beta(C \times D, C \times D) \\
& \text { Note that } \beta(A \times B, A \times D)=0 \text { by the definition of } \tilde{\beta} \\
& =\beta(A \times B, A \times B)+\beta(A \times D, A \times D)+\beta(C \times B, C \times B)+\beta(C \times D, C \times D) \\
& =\tilde{\beta}(A, B)+\tilde{\beta}(A, D)+\tilde{\beta}(C, B)+\tilde{\beta}(C, D)
\end{aligned}
$$

Since $\beta$ is $\sigma$-additive, $\tilde{\beta}$ is also $\sigma$-additive. 
We have now well-defined a product of two positive definite bimeasures. We also define O-dot product of random measures $Z_{1}$ and $Z_{2}$, which are corresponding random measures of $\beta_{1}, \beta_{2}$ by Lemma 2.3 .

Definition 3.2 Suppose the $\beta=\beta_{1} \cdot \beta_{2}:(\mathcal{G} \times \mathcal{G}) \times(\mathcal{G} \times \mathcal{G}) \rightarrow \mathbb{C}$ as in Definition 2.7. Let $\tilde{\beta}=\beta$ on the diagonal of $(\mathcal{G} \times \mathcal{G}) \times(\mathcal{G} \times \mathcal{G})$, and 0 otherwise. Define $O$-dot product $\odot$ of bimeasures by $\tilde{\beta}=\beta_{1} \odot \beta_{2}$. Therefore, $\tilde{\beta}: \mathcal{G} \times \mathcal{G} \rightarrow \mathbb{C}$ is defined by $\tilde{\beta}(A, B)=\beta_{1} \odot \beta_{2}(A, B)=\beta_{1}(A, B) \cdot \beta_{2}(A, B)$, where $A \times B \in \mathcal{G} \times \mathcal{G}$. Moreover, there exist a reproducing kernel Hilbert space, $\mathbb{H}$ of $\tilde{\beta}$ and a random measure $Z$ such that $\tilde{\beta}(A, B)=E[Z(A) \overline{Z(B)}]$. If $Z_{1}, Z_{2}$ and $\mathbb{H}_{1}, \mathbb{H}_{2}$ are the corresponding random measures and reproducing kernel Hilbert spaces for bimeasures $\beta_{1}, \beta_{2}$, then define the $O$-dot product of $Z_{1}$ and $Z_{2}$ as $Z=Z_{1} \odot Z_{2}$, whose bimeasure is $\tilde{\beta}$.

Remark 2 Note that there is a slight change from the definition of O-dot product in Rao (2012). In this article, we have restricted domain of the product bimeasure $\beta$ so it can have the same domain of $\beta_{1}, \beta_{2}$.

3.2 Structure of $B M(G, \odot)$ and $R M(G, \odot)$

We construct an algebra of random measures under O-dot product finally. If $Z=Z_{1} \odot Z_{2}$ and $\beta_{1}, \beta_{2}$ are corresponding bimeasures of $Z_{1}, Z_{2}$, respectively, then we will denote $\beta$ as the corresponding bimeasure of $Z$ and $\beta=\beta_{1} \odot \beta_{2}$. Note that we omit the tilde notation ( $\sim$ ) above $\beta$ for convenience of writing notations.

Theorem 3.3 $B M(G, \odot)$ is a ring.

Proof i) $B M(G,+)$ is an abelian group by Theorem 2.5. ii) We show the associative property. Let $A \times B \in \mathcal{G} \times \mathcal{G}$. $\beta_{1} \odot\left(\beta_{2} \odot \beta_{3}\right)(A, B)=\beta_{1}(A, B) \cdot\left(\beta_{2} \odot \beta_{3}(A, B)\right)=\beta_{1}(A, B) \cdot\left(\beta_{2}(A, B) \cdot \beta_{3}(A, B)\right)=\beta_{1}(A, B) \cdot \beta_{2}(A, B) \cdot \beta_{3}(A, B)=$ $\left(\beta_{1} \odot \beta_{2}(A, B)\right) \cdot \beta_{3}(A, B)=\left(\beta_{1} \odot \beta_{2}\right) \odot \beta_{3}(A, B)$ iii) We show the distributive property. $\beta_{1} \odot\left(\beta_{2}+\beta_{3}\right)(A, B)=\beta_{1}(A, B) \cdot\left(\beta_{2}+\right.$ $\left.\beta_{3}\right)(A, B)=\beta_{1}(A, B) \cdot\left(\beta_{2}(A, B)+\beta_{3}(A, B)\right)=\beta_{1}(A, B) \cdot \beta_{2}(A, B)+\beta_{1}(A, B) \cdot \beta_{3}(A, B)=\left(\beta_{1} \odot \beta_{2}\right)(A, B)+\left(\beta_{1} \odot \beta_{3}\right)(A, B)$.

Remark 3 The multiplicative identity of $B M(G)$ is not trivial. One can think of a bimeasure $\delta(A, B)=1$ for all $A, B \in \mathcal{G}$. However, this $\delta$ will not have the additive property of bimeasure.

Theorem 3.4 $B M(G, \odot)$ is an algebra over $\mathbb{C}$.

Proof i) $B M(G)$ is a unitary $\mathbb{C}$-module by Theorem 2.5. ii) Let $a \in \mathbb{C}$. Then $a\left(\beta_{1} \odot \beta_{2}\right)(A, B)=a\left(\beta_{1}(A, B) \cdot \beta_{2}(A, B)\right)=$ $a \beta_{1}(A, B) \cdot \beta_{2}(A, B)=\left(a \beta_{1} \odot \beta_{2}\right)(A, B)=\beta_{1}(A, B) \cdot a \beta_{2}(A, B)=\left(\beta_{1} \odot a \beta_{2}\right)(A, B)$.

Theorem 3.5 $R M(G, \odot)$ is a ring.

Proof Let $Z_{1}, Z_{2}, Z_{3} \in R M(G)$, and $\beta_{1}, \beta_{2}, \beta_{3}$ be their corresponding bimeasures. (i.e. $Z_{1} \sim \beta_{1}, Z_{2} \sim \beta_{2}, Z_{3} \sim \beta_{3}$ ). i) $R M(G,+)$ is an abelian group by Theorem 2.5. ii) Note $Z_{1} \odot Z_{2} \sim \beta_{1} \odot \beta_{2}$ by definition. $\left(Z_{1} \odot Z_{2}\right) \odot Z_{3} \sim\left(\beta_{1} \odot \beta_{2}\right) \odot \beta_{3}=$ $\beta_{1} \odot\left(\beta_{2} \odot \beta_{3}\right) \sim Z_{1} \odot\left(Z_{2} \odot Z_{3}\right)$. iii) $Z_{1} \odot\left(Z_{2}+Z_{3}\right) \sim \beta_{1} \odot\left(\beta_{2}+\beta_{3}\right)=\beta_{1} \odot \beta_{2}+\beta_{1} \odot \beta_{3} \sim Z_{1} \odot Z_{2}+Z_{1} \odot Z_{3}$. iv $)$ $Z_{1} \odot Z_{2} \sim \beta_{1} \odot \beta_{2}=\beta_{2} \odot \beta_{1} \sim Z_{2} \odot Z_{1}$.

Theorem 3.6 $R M(G, \odot)$ is an algebra over $\mathbb{C}$.

Proof Let $Z_{1}, Z_{2} \in R M(G)$ and $k \in \mathbb{C}$. Then i) $R M(G,+)$ is a unitary $\mathbb{C}$-module by Theorem 2.5. ii) Recall $k Z \sim|k|^{2} \beta$ by Lemma 2.4. $k\left(Z_{1} \odot Z_{2}\right) \sim|k|^{2}\left(\beta_{1} \odot \beta_{2}\right)=|k|^{2} \beta_{1} \odot \beta_{2}=\beta_{1} \odot|k|^{2} \beta_{2} \sim k Z_{1} \odot Z_{2}$ and $\sim Z_{1} \odot k Z_{2}$, respectively.

\subsection{O-dot Product of Wiener Processes}

In this section, $\mathrm{O}$-dot product of bimeasures of Wiener processes is considered as an explicit example.

Lemma 3.7 Suppose $Z_{W}: \mathcal{G} \rightarrow L^{2}(P)$ is a random measure that represesnts Wiener Process, where $\mathcal{G}$ is a $\sigma$-algebra of Borel subsets of $\mathbb{R}^{+}$. Suppose $\beta_{W}$ is the corresponding bimeasure of $Z_{W}$ (i.e. $\beta_{W}$ is a scalar bimeasure induced from a Wiener process). If $Z=Z_{W} \odot Z_{W}: \mathcal{G} \rightarrow L^{2}(P)$, then $Z$ has the bimeasure $\beta=\beta_{W} \odot \beta_{W}: \mathcal{G} \times \mathcal{G} \rightarrow \mathbb{C}$ such that $\beta(A, B)=\sigma^{4} \mu(A \cap B)^{2}$, where $\sigma$ is a positive diffusion coefficient of the Wiener Process.

Proof By the definition of $\beta$,

$$
\begin{aligned}
\beta(A, B) & =\beta_{W} \odot \beta_{W}(A, B) \\
& =\beta_{W}(A, B) \cdot \beta_{W}(A, B) \\
& =\sigma^{2} \mu(A \cap B) \cdot \sigma^{2} \mu(A \cap B) \\
& =\sigma^{4} \mu(A \cap B)^{2} .
\end{aligned}
$$

$Z \odot Z$ has the covariance bimeasure $\beta=\sigma^{4}(\mu(A \cap B))^{2}$, where $Z$ is a random measure of Wiener process. There exist a unique Gaussian Process corresponding to a given bimeasure. However, $Z \odot Z$ itself will not be the random measure of Wiener process.

\section{Convolution by Strict Morse-Transue Integral}

In this section, we will illustrate the random measure algebra with the convolution by Morse-Transue Integral as in 
Proposition 2.9. The definition of convolution by Morse-Transue Integral is well-defined in Rao (2012). The following lemma shows the convolution is commutative.

Lemma 4.1 The convolution product of positive definite bimeasures is commutative.

Proof

$$
\begin{aligned}
\beta_{1} * \beta_{2}(A, B) & =\int_{0}^{1} \int_{0}^{1} \beta_{1}(A-x, B-y) d \beta_{2}(x, y) \\
& =\int_{0}^{1} \int_{0}^{1} \int_{0}^{1} \int_{0}^{1} \chi_{A, B}(a+x, b+y) d \beta_{1}(a, b) d \beta_{2}(x, y) \\
& =\int_{0}^{1} \int_{0}^{1} \int_{0}^{1} \int_{0}^{1} \chi_{A, B}(a+x, b+y) d \beta_{2}(x, y) d \beta_{1}(a, b) \\
& =\int_{0}^{1} \int_{0}^{1} \beta_{2}(A-a, B-b) d \beta_{1}(a, b) \\
& =\beta_{2} * \beta_{1}(A, B)
\end{aligned}
$$

\subsection{Structure of $B M([0,1])$}

Let's denote $B M([0,1])$ as a set of positive definite bimeasures $\beta: \mathcal{B}([0,1]) \times \mathcal{B}([0,1]) \rightarrow \mathbb{C}$, where $\mathcal{B}([0,1])$ is a $\sigma$-algebra of Borel subsets of $[0,1]$, and denote $R M([0,1])$ as a set of random measures $Z: \mathcal{B}([0,1]) \rightarrow L^{2}(\Omega, \Sigma, P)$. We investigate the algebraic structure of $B M([0,1])$ and $R M([0,1])$ with the convolution.

Theorem 4.2 The set of bimeasure BM([0,1]) with the convolution by Morse-Transue Integral is a ring with identity.

Proof It is trivial to show that $B M([0,1])$ is a group under addition. We show that $B M([0,1])$ is a monoid space under convolution $*$ and has the distributive property.

First, we want to show $\left(\beta_{1} * \beta_{2}\right) * \beta_{3}=\beta_{1} *\left(\beta_{2} * \beta_{3}\right)$. We use Fubini's Theorem for bimeasures and commutative properties.

$$
\begin{aligned}
\left(\left(\beta_{1} * \beta_{2}\right) * \beta_{3}\right)(A, B) & =\int_{0}^{1} \int_{0}^{1} \beta_{1} * \beta_{2}(A-x, B-y) \beta_{3}(d x, d y) \\
& =\int_{0}^{1} \int_{0}^{1} \int_{0}^{1} \int_{0}^{1} \beta_{1}(A-x-a, B-y-b) d \beta_{2}(a, b) d \beta_{3}(x, y) \\
& =\int_{0}^{1} \int_{0}^{1} \int_{0}^{1} \int_{0}^{1} \beta_{1}(A-x-a, B-y-b) d \beta_{3}(x, y) d \beta_{2}(a, b) \\
& =\int_{0}^{1} \int_{0}^{1} \int_{0}^{1} \int_{0}^{1} \beta_{3}(A-x-a, B-y-b) d \beta_{1}(x, y) d \beta_{2}(a, b) \\
& =\int_{0}^{1} \int_{0}^{1} \int_{0}^{1} \int_{0}^{1} \beta_{3}(A-x-a, B-y-b) d \beta_{2}(a, b) d \beta_{1}(x, y) \\
& =\int_{0}^{1} \int_{0}^{1} \beta_{3} * \beta_{2}(A-x, B-y) d \beta_{1}(x, y) \\
& =\left(\left(\beta_{3} * \beta_{2} * \beta_{1}\right)(A, B)\right. \\
& =\left(\beta_{1} *\left(\beta_{3} * \beta_{2}\right)\right)(A, B)=\left(\beta_{1} *\left(\beta_{2} * \beta_{3}\right)\right)(A, B)
\end{aligned}
$$

Simliarly, one can show $\left(\beta_{1} *\left(\beta_{3} * \beta_{2}\right)\right)(A, B)=\left(\beta_{1} *\left(\beta_{2} * \beta_{3}\right)\right)(A, B)$.

$B M([0,1])$ has a unit $\delta_{0}(\cdot, \cdot)$, where $\delta_{0}(\cdot, \cdot)$ is defined by

$$
\delta_{0}(A, B)= \begin{cases}1 & \text { if } 0 \in A \text { and } 0 \in B \\ 0 & \text { otherwise }\end{cases}
$$

Observe that $\delta_{0}$ is a bimeasure since it has the $\sigma$-additive property $\delta_{0}\left(A, \cup_{n \in I} B_{n}\right)=\Sigma_{n \in I} \delta_{0}\left(A, B_{n}\right)$. Also $\beta * \delta_{0}(A, B)=$ $\int_{0}^{1} \int_{0}^{1} \beta(A-x, B-y) d \delta_{0}(d x, d y)=\beta(A, B)$, and $\delta_{0} * \beta(A, B)=\int_{0}^{1} \int_{0}^{1} \delta_{0}(A-a, B-b) d \beta(a, b)=\beta(A, B)$.

For the multiplicative distributive property, we have

$$
\begin{aligned}
\beta_{1} *\left(\beta_{2}+\beta_{3}\right)(A, B) & =\int_{0}^{1} \int_{0}^{1} \beta_{1}(A-x, B-y) d\left(\beta_{2}+\beta_{3}\right)(x, y) \\
& =\int_{0}^{1} \int_{0}^{1} \beta_{1}(A-x, B-y) d \beta_{2}+\int_{0}^{1} \int_{0}^{1} \beta_{1}(A-x, B-y) d \beta_{3} \\
& =\left(\beta_{1} * \beta_{2}+\beta_{1} * \beta_{3}\right)(A, B)
\end{aligned}
$$

Therefore, $B M[0,1]$ is a ring.

We extend the structure to $\mathbb{C}$-algebra.

Theorem 4.3 $B M([0,1]$, *) is a $\mathbb{C}$-algebra.

Proof $B M([0,1],+)$ is a $\mathbb{C}$-module. We have the compatibility with scalars, that is $\left(a \beta_{1}\right) *\left(b \beta_{2}\right)(A, B)=\int_{0}^{1} \int_{0}^{1} a \beta_{1}(A-$ $x, B-y) d\left(b \beta_{2}\right)(x, y)=a b \int_{0}^{1} \int_{0}^{1} \beta_{1}(A-x, B-y) d \beta_{2}(x, y)=a b \beta_{1} * \beta_{2}(A, B)$.

4.2 Structure of $R M([0,1]$, *)

We move on to the structure of random measure algebra, which is our main interest. 
Definition 4.4 Given the Borel measurable space $([0,1], \mathcal{B}([0,1]))$, let $Z_{i}: \mathcal{B}([0,1]) \rightarrow L_{0}^{2}(P)$ be a pair $(i=1,2)$ of random measures into the Hilbert space of (equivalence classes of) centered (complex) random variables on a probability space $(\Omega, \Sigma, P)$ with covariance bimeasures $\beta_{i}: \mathcal{B}([0,1]) \times \mathcal{B}([0,1]) \rightarrow \mathbb{C}$ given by $\beta_{i}(A, B)=<Z_{i}(A), Z_{i}(B)>$ using the inner product notation. Let $\beta=\beta_{1} * \beta_{2}: \mathcal{B}([0,1]) \times \mathcal{B}([0,1]) \rightarrow \mathbb{C}$ be the convolution product given in Proposition 2.9. If $Z: \mathcal{B}([0,1]) \rightarrow L_{0}^{2}(P)$ is the induced random measure by $\beta$, then denote by $Z=Z_{1} * Z_{2}: \mathcal{B}([0,1]) \rightarrow L_{0}^{2}(P)$, the random measure. It is well defined as convolution of $Z_{1}$ and $Z_{2}$.

The convolution of the bimeasures is the key to define the convolution of random measures, since a positive definite bimeasure induces a random measure, and vice versa, by Lemma 2.3.

Theorem $4.5 \operatorname{RM}([0,1], *)$ is a ring with identity.

Proof i) $R M([0,1], *)$ is an abelian group. ii) $Z_{1} *\left(Z_{2}+Z_{3}\right) \sim \beta_{1} *\left(\beta_{2}+\beta_{3}\right)=\beta_{1} * \beta_{2}+\beta_{1}+\beta_{3} \sim Z_{1} * Z_{2}+Z_{1} * Z_{3}$. iii) $\left(Z_{1}+Z_{2}\right) * Z_{3} \sim\left(\beta_{1}+\beta_{2}\right) * \beta_{3}=\beta_{1} * \beta_{3}+\beta_{1} * \beta_{3} \sim Z_{1} * Z_{3}+Z_{2} * Z_{3}$. iv) $\left(Z_{1} * Z_{2}\right) * Z_{3} \sim\left(\beta_{1} * \beta_{2}\right) * \beta_{3}=\beta_{1} *\left(\beta_{2} * \beta_{3}\right) \sim Z_{1} *\left(Z_{2} * Z_{3}\right)$. v) $Z_{1} * Z_{2} \sim Z_{2} * Z_{1}$ for commutativity. vi) Define a random measure $Z_{0}(E)=1$ if $0 \in E$, 1 otherwise. $Z_{0}$ is finitely additive since $Z_{0}\left(\cup E_{n}\right)=\Sigma Z_{0}\left(E_{n}\right)=1$ if $0 \in E_{i}$ for any $i, 0$ otherwise. Let $\delta_{0}$ be correspoding bimeasure of $Z_{0}$. Then $\delta_{0}(A, B)=E[Z(A) Z(B)]=1$ if $0 \in A$ and $0 \in B, 0$ otherwise, which is the exact definition of Dirac $\delta$-function. (i.e $Z_{0} \sim \delta_{0} . Z_{1} * Z_{0} \sim \beta_{1} * \delta_{0}=\beta_{1} \sim Z_{1}$. Similarly, $Z_{0} * Z_{1} \sim Z_{1}$.

Theorem 4.6 $R M([0,1]$, *) is a $\mathbb{C}$-algebra.

Proof i) $R M([0,1])$ is a $\mathbb{C}$-module. ii) $k\left(Z_{1} * Z_{2}\right) \sim|k|^{2}\left(\beta_{1} * \beta_{2}\right)=|k|^{2} \beta_{1} * \beta_{2}=\beta_{1} *|k|^{2} \beta_{2} \sim k Z_{1} * Z_{2}$ and $\sim Z_{1} * k Z_{2}$.

Finally, an algebra of random measure is constructed.

\subsection{Wiener Process}

Let $\left\{W_{t}\right\}_{t \in[0,1]}$ be a Wiener Process, and $Z$ be the associated random measure, that is $Z: \mathcal{B}([0,1]) \rightarrow L^{2}(\Omega, \Sigma, P)$ such that $Z\left(\left[t_{i}, t_{i+1}\right]\right)=W_{t_{i}}-W_{t_{i+1}}$. Let $\beta$ be the bimeasure of $Z$, that is $\beta(A, B)=E[Z(A) \overline{Z(B)}]$. We explicitly compute $\beta * \beta(A, B)$ if $A=\left[t_{1}, t_{2}\right], B=\left[s_{1}, s_{2}\right]$ with $s_{1}<t_{1}<s_{2}<t_{2}$.

Theorem 4.7 Let $\left\{W_{t}\right\}_{t \in[0,1]}$ be a Wiener process, and $Z$ be the associated random measure, that is $Z: \mathcal{B}([0,1]) \rightarrow$ $L^{2}(\Omega, \Sigma, P)$ such that $Z\left(\left[t_{i}, t_{i+1}\right]\right)=W_{t_{i}}-W_{t_{i+1}}$. Let $\beta$ be the bimeasure of $Z$, that is $\beta(A, B)=E[Z(A) \overline{Z(B)}]$ with $A=$ $\left[t_{1}, t_{2}\right], B=\left[s_{1}, s_{2}\right]$ with $s_{1}<t_{1}<s_{2}<t_{2}$. Then

$$
\begin{aligned}
\beta * \beta(A, B) & =-\frac{s_{1}^{2} t_{1}^{2}}{4}+\frac{s_{2} t_{1}^{3}}{4}-\frac{t_{1}^{4}}{12}+\frac{s_{2}}{3}+\frac{t_{1} s_{2}}{2}+\frac{t_{1}^{2} s_{2}}{2}+\frac{t_{1}^{3} s_{2}}{6}-\frac{s_{2}^{2}}{2}-\frac{t_{1} s_{2}^{2}}{2}-\frac{t_{1}^{2} s_{2}^{2}}{4} \\
& +\frac{t_{1} s_{2}^{2}}{6}-\frac{s^{4}}{12}+\frac{t_{2}}{3}+\frac{s_{1} t_{2}}{2}+\frac{s_{1}^{2} t_{2}}{2}+\frac{s_{1}^{3} t_{2}}{6}+\frac{s_{2} t_{2}}{2}+\frac{s_{2}^{2} t_{2}}{2}+\frac{s_{2}^{3} t_{2}}{6}-\frac{t_{2}^{2}}{2} \\
& -\frac{s_{1} t_{2}^{2}}{2}-\frac{s_{1}^{2} t_{2}^{2}}{4}-\frac{s_{2} t_{2}^{2}}{2}-\frac{s_{2}^{2} t_{2}^{2}}{4}+\frac{t_{2}^{3}}{3}+\frac{s_{1} t_{2}^{3}}{6}+\frac{s_{2} t_{2}^{3}}{6}-\frac{t_{2}^{4}}{12} .
\end{aligned}
$$

Proof We are to compute

$$
\beta_{1} * \beta_{2}(A, B)=\int_{0}^{1} \int_{0}^{1} \beta_{1}(A-x, B-y) \beta_{2}(d x, d y), \quad \text { with } A, B \in \mathcal{B}([0,1])
$$

where $\beta_{1}, \beta_{2}$ are both bimeasures of random measures from Wiener process. We investigate $\beta(A-x, B-y)$ by,

$$
\begin{aligned}
\beta(A-x, B-y) & =E[Z(A-x) Z(B-y)] \text { where } Z(\cdot) \text { is as above } \\
& =E\left[\left(X\left(t_{2}-x\right)-X\left(t_{1}-x\right)\right) \cdot\left(X\left(s_{2}-y\right)-X\left(s_{1}-y\right)\right)\right] \\
& =E\left[X\left(t_{2}-x\right) X\left(s_{2}-y\right)\right]-E\left[X\left(t_{2}-x\right) X\left(s_{1}-y\right)\right] \\
& -E\left[X\left(t_{1}-x\right) X\left(s_{2}-y\right)\right]+E\left[X\left(t_{1}-x\right) X\left(s_{1}-y\right)\right] \\
& =\min \left\{t_{2}-x, s_{2}-y\right\}-\min \left\{t_{2}-x, s_{1}-y\right\} \\
& -\min \left\{t_{1}-x, s_{2}-y\right\}+\min \left\{t_{1}-x, s_{1}-y\right\} \\
& \text { by Lemma } 2.12 .
\end{aligned}
$$

Therefore,

$$
\begin{aligned}
\beta_{1} * \beta_{2}(A, B) & =\int_{0}^{1} \int_{0}^{1} \beta_{1}(A-x, B-y) \beta_{2}(d x, d y) \\
& =\int_{0}^{1} \int_{0}^{1} \min \left\{t_{2}-x, s_{2}-y\right\}-\min \left\{t_{2}-x, s_{1}-y\right\} \\
& -\min \left\{t_{1}-x, s_{2}-y\right\}+\min \left\{t_{1}-x, s_{1}-y\right\} d \beta_{2}(x, y) \\
& =\int_{0}^{1} \int_{0}^{1} \min \left\{t_{2}-x, s_{2}-y\right\} d \beta_{2}(x, y) \\
& -\int_{0}^{1} \int_{0}^{1} \min \left\{t_{2}-x, s_{1}-y\right\} d \beta_{2}(x, y) \\
& -\int_{0}^{1} \int_{0}^{1} \min \left\{t_{1}-x, s_{2}-y\right\} d \beta_{2}(x, y) \\
& +\int_{0}^{1} \int_{0}^{1} \min \left\{t_{1}-x, s_{1}-y\right\} d \beta_{2}(x, y)
\end{aligned}
$$


Observe that

$$
\begin{aligned}
& \min \left\{t_{2}-x, s_{2}-y\right\}= \begin{cases}t_{2}-x & \text { if } x-\left(t_{2}-s_{2}\right) \geq y \\
s_{2}-y & \text { if } x-\left(t_{2}-s_{2}\right)<y\end{cases} \\
& \min \left\{t_{2}-x, s_{1}-y\right\}= \begin{cases}t_{2}-x & \text { if } x-\left(t_{2}-s_{1}\right) \geq y \\
s_{1}-y & \text { if } x-\left(t_{2}-s_{1}\right)<y\end{cases} \\
& \min \left\{t_{1}-x, s_{2}-y\right\}= \begin{cases}t_{1}-x & \text { if } x+s_{2}-t_{1} \geq y \\
s_{2}-y & \text { if } x+s_{2}-t_{1}<y\end{cases} \\
& \min \left\{t_{1}-x, s_{1}-y\right\}= \begin{cases}t_{1}-x & \text { if } x-\left(t_{1}-s_{1}\right) \geq y \\
s_{1}-y & \text { if } x-\left(t_{1}-s_{1}\right)<y\end{cases}
\end{aligned}
$$

Also $d \beta(x, y)=d(\min (x, y))=x d x d y$ if $x \leq y$, and $=y d x d y$ if $x>y$. We will now calculate each of the integrals explicitly.

$$
\begin{aligned}
& \int_{0}^{1} \int_{0}^{1} \min \left\{t_{2}-x, s_{2}-y\right\} d \beta_{2}(x, y) \\
& =\int_{0}^{1} \int_{0}^{y} x\left(s_{2}-y\right) d x d y+\int_{0}^{t_{2}-s_{2}} \int_{y}^{t_{2}-s_{2}} y\left(s_{2}-y\right) d x d y \\
& +\int_{t_{2}-s_{2}}^{1} \int_{x-\left(t_{2}-s_{2}\right)}^{x} y\left(s_{2}-y\right) d y d x+\int_{t_{2}-s_{2}}^{1} \int_{0}^{x-\left(t_{2}-s_{2}\right)} y\left(t_{2}-x\right) d y d x \\
& =-\frac{1}{8}+\frac{s_{2}}{6} \\
& -\frac{1}{12}\left(s_{2}-t_{2}\right)^{3}\left(3 s_{2}-t_{2}\right) \\
& +\frac{1}{6}\left(s_{2}^{4}-s_{2}^{3}\left(4 t_{2}+1\right)+6 s_{2}^{2} t_{2}^{2}+s_{2}\left(-4 t_{2}^{3}+3 t_{2}^{2}-3 t_{2}+2\right)+t_{2}\left(t_{2}^{3}-2 t_{2}^{2}+3 t_{2}-2\right)\right) \\
& +\frac{1}{24}\left(s_{2}-t_{2}+1\right)^{3}\left(s_{2}+3 t_{2}-3\right)
\end{aligned}
$$

Similarly, other integrals can be obtained. Thus,

$$
\begin{aligned}
& \int_{0}^{1} \int_{0}^{1} \min \left\{t_{2}-x, s_{1}-y\right\} d \beta_{2}(x, y) \\
& =\int_{0}^{1} \int_{0}^{y} x\left(s_{1}-y\right) d x d y+\int_{0}^{t_{2}-s_{1}} \int_{y}^{t_{2}-s_{1}} y\left(s_{1}-y\right) d x d y \\
& +\int_{t_{2}-s_{1}}^{1} \int_{x-\left(t_{2}-s_{1}\right)}^{x} y\left(s_{1}-y\right) d y d x+\int_{t_{2}-s_{1}}^{1} \int_{0}^{x-\left(t_{2}-s_{1}\right)} y\left(t_{2}-x\right) d y d x \\
& =-\frac{1}{8}+\frac{s_{1}}{6} \\
& -\frac{1}{1^{2}}\left(s_{1}-t_{2}\right)^{3}\left(3 s_{1}-t_{2}\right) \\
& +\frac{1}{6}\left(s_{1}^{4}-s_{1}^{3}\left(4 t_{2}+1\right)+6 s_{1}^{2} t_{2}^{2}+s_{1}\left(-4 t_{2}^{3}+3 t_{2}^{2}-3 t_{2}+2\right)+t_{2}\left(t_{2}^{3}-2 t_{2}^{2}+3 t_{2}-2\right)\right) \\
& +\frac{1}{24}\left(s_{1}+3 t_{2}-3\right)\left(s_{1}-t_{2}+1\right)^{3}
\end{aligned}
$$

and

$$
\begin{aligned}
& \int_{0}^{1} \int_{0}^{1} \min \left\{t_{1}-x, s_{2}-y\right\} d \beta_{2}(x, y) \\
& =\int_{s_{2}-t_{1}}^{1} \int_{0}^{y-s_{2}+t_{1}} x\left(s_{2}-y\right) d x d y+\int_{0}^{1-s_{2}+t_{1}} \int_{x}^{x+s_{2}-t_{1}} x\left(t_{1}-x\right) d y d x \\
& +\int_{1-s_{2}+t_{1}}^{1} \int_{x}^{1} x\left(t_{1}-x\right) d y d x+\int_{0}^{1} \int_{0}^{x} y\left(t_{1}-x\right) d y d x \\
& =\frac{1}{24}\left(1+t_{1}-s_{2}\right)^{3}\left(-3+t_{1}+3 s_{2}\right)+\frac{1}{6}\left(1+t_{1}-s_{2}\right)^{2}\left(-t_{1}+s_{2}\right)\left(-2+t_{1}+2 s_{2}\right) \\
& +\frac{1}{12}\left(t_{1}-s_{2}\right)^{2}\left(-6+t_{1}^{2}+2 t_{1}\left(-1+s_{2}\right)+8 s_{2}-3 s_{2}^{2}\right) \\
& +\frac{t_{1}}{6}-\frac{1}{8}
\end{aligned}
$$

The last integral is

$$
\begin{aligned}
& \int_{0}^{1} \int_{0}^{1} \min \left\{t_{1}-x, s_{1}-y\right\} d \beta_{2}(x, y) \\
& =\int_{0}^{1} \int_{0}^{y} x\left(s_{1}-y\right) d x d y+\int_{0}^{t_{1}-s_{1}} \int_{y}^{t_{1}-s_{1}} y\left(s_{1}-y\right) d x d y \\
& +\int_{t_{1}-s_{1}}^{1} \int_{x-\left(t_{1}-s_{1}\right)}^{x} y\left(s_{1}-y\right) d y d x+\int_{t_{1}-s_{1}}^{1} \int_{0}^{x-\left(t_{1}-s_{1}\right)} y\left(t_{1}-x\right) d y d x \\
& =-\frac{1}{8}+\frac{s_{1}}{6} \\
& -\frac{1}{12}\left(s_{1}-t_{1}\right)^{3}\left(3 s_{1}-t_{1}\right) \\
& +\frac{1}{6}\left(s_{1}^{4}-s_{1}^{3}\left(4 t_{1}+1\right)+6 s_{1}^{2} t_{1}^{2}+s_{1}\left(-4 t_{1}^{3}+3 t_{1}^{2}-3 t_{1}+2\right)+t_{1}\left(t_{1}^{3}-2 t_{1}^{2}+3 t_{1}-2\right)\right) \\
& +\frac{1}{24}\left(s_{1}+3 t_{1}-3\right)\left(s_{1}-t_{1}+1\right)^{3}
\end{aligned}
$$


We put these calculated integrals together.

$$
\begin{aligned}
\beta_{1} * \beta_{2}(A, B) & =\int_{0}^{1} \int_{0}^{1} \beta_{1}(A-x, B-y) \beta_{2}(d x, d y) \\
& =\int_{0}^{1} \int_{0}^{1} \min \left\{t_{2}-x, s_{2}-y\right\} d \beta_{2}(x, y) \\
& -\int_{0}^{1} \int_{0}^{1} \min \left\{t_{2}-x, s_{1}-y\right\} d \beta_{2}(x, y) \\
& -\int_{0}^{1} \int_{0}^{1} \min \left\{t_{1}-x, s_{2}-y\right\} d \beta_{2}(x, y) \\
& +\int_{0}^{1} \int_{0}^{1} \min \left\{t_{1}-x, s_{1}-y\right\} d \beta_{2}(x, y) \\
& =-1+\frac{s_{1}}{3}-\frac{s_{1}^{2}}{2}-\frac{s_{1}^{3}}{3}-\frac{s_{1}^{4}}{12}+\frac{t_{1}}{3}+\frac{s_{1} t_{1}}{2}+\frac{s_{1}^{2} t_{1}}{2}+\frac{s_{1}^{3} t_{1}}{6}-\frac{t_{1}^{2}}{2}-\frac{s_{1} t_{1}^{2}}{2} \\
& -\frac{s_{1}^{2} t_{1}^{2}}{4}+\frac{s_{2} t_{1}^{3}}{4}-\frac{t_{1}^{4}}{12}+\frac{s_{2}}{3}+\frac{t_{1} s_{2}}{2}+\frac{t_{1}^{2} s_{2}}{2}+\frac{t_{1}^{3} s_{2}}{6}-\frac{s_{2}^{2}}{2}-\frac{t_{1} s_{2}^{2}}{2}-\frac{t_{1}^{2} s_{2}^{2}}{4} \\
& +\frac{t_{1} s_{2}^{3}}{6}-\frac{s^{4}}{12}+\frac{t_{2}}{3}+\frac{s_{1} t_{2}}{2}+\frac{s_{1}^{2} t_{2}}{2}+\frac{s_{1}^{3} t_{2}}{6}+\frac{s_{2} t_{2}}{2}+\frac{s_{2}^{2} t_{2}}{2}+\frac{s_{2}^{3} t_{2}}{6}-\frac{t_{2}^{2}}{2} \\
& -\frac{s_{1} t_{2}^{2}}{2}-\frac{s_{1}^{2} t_{2}^{2}}{4}-\frac{s_{2} t_{2}^{2}}{2}-\frac{s_{2}^{2} t_{2}}{4}+\frac{t_{2}^{3}}{3}+\frac{s_{1} t_{2}^{3}}{6}+\frac{s_{2} t_{2}^{3}}{6}-\frac{t_{2}^{4}}{12}
\end{aligned}
$$

There exists a unique (centered) Gaussian Process corresponding to a given covariance function and hence for that we just computed, since it is well known that a Gaussian process is determined by its mean and covariance functions. However, with such a complex representation, it is not trivial to express the exact representation of the Gasussian Process related to the covariance function above, although there exists a unique (Gaussian) process, which will not be the (traditional) Wiener process.

\section{Acknowledgements}

This paper forms part of a dissertation submitted to the University of California, Riverside in partial fulfillment of the requirements for the Ph.D. degree. I would like to express my very great appreciation to my advisor Professor M.M. Rao.

\section{References}

Chang, J., Chung, H. S., \& Skoug, D. (2014). Relationships Involving Transforms and Convolutions Via the Translation Theorem. Stochastic Analysis and Application, 32(2), 348-363. https://doi.org/10.1080/07362994.2013.877350

Dehay, D. (1991). On the Product of Two Harmonizable Time Series. Stochastic Processes and their Applications, 38, 347-358. https://doi.org/10.1016/0304-4149(91)90099-X

Dunford, N., \& Schwarz, J. T. (1958). Linear Operators, Part I: General Theory. New York, NY: Interscience.

Graham, C., \& Schreiber, B. (1984). Bimeasure Algebras on LCA Groups. Pacific Journal of Mathematics, 115, 91-127. https://doi.org/10.2140/pjm.1984.115.91

Huneycutt, J. E. (1972). Products and Convolutions of Vector-Valued Set Functions. Studia Math., 41, 119-129. https://doi.org/10.4064/sm-41-2-119-129

Morse, M., \& Transue, W. (1956). C-Bimeasures and Their Integral Extensions. Ann. of Math., 64, 480-504. https://doi.org/10.2307/1969597

Park, J. H. J. (2016). A Random Measure Alegebra under Convolution. Journal of Statistical Theory and Practice, 10(4), 768-779. https://doi.org/10.1080/15598608.2016.1224745

Park, J. H. J. (2015). Random Measure Algebras under Convolution. Riverside, CA: Ph.D. Thesis, UCR Library.

Rao, M. M. (2012). Random and Vector Measures. Singapore: World Scientific. https://doi.org/10.1142/9789814350822

\section{Copyrights}

Copyright for this article is retained by the author(s), with first publication rights granted to the journal.

This is an open-access article distributed under the terms and conditions of the Creative Commons Attribution license (http://creativecommons.org/licenses/by/4.0/). 